
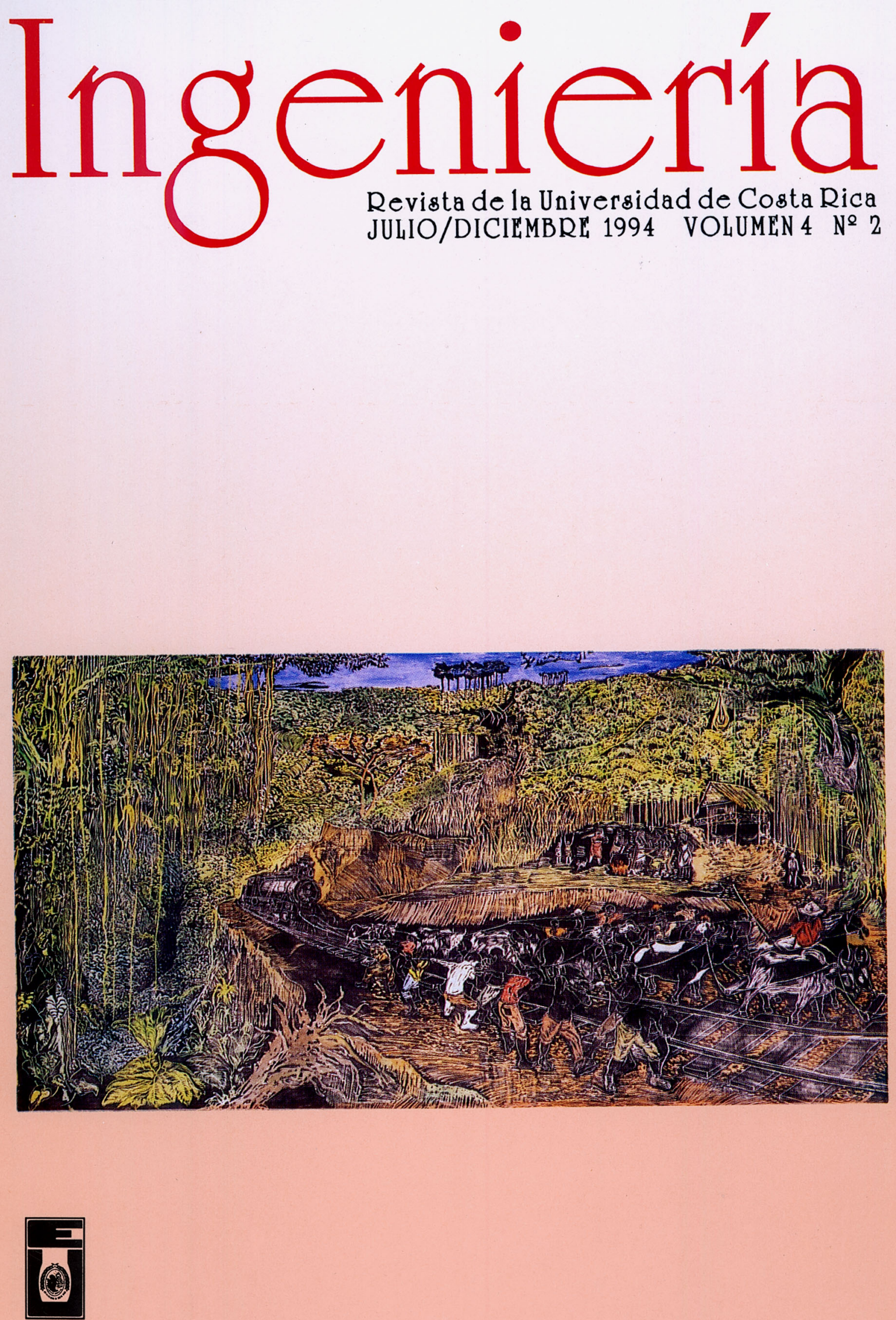


\title{
FUNCIÓN DEL POSPROCESADOR EN UN SISTEMA CAD/CAM
}

\author{
Ing. Luis Arturo Rapso,Msc.
}

\begin{abstract}
RESUMEN
El presente trabajo tiene como objetivo dar una descripción concisa de la función del sistema posprocesador dentro de un sistema CAD/CAM. Son presentadas dos concepciones diferentes de posprocesadores, uno de ellos de carácter general, orientado al uso con procesadores tipo APT o semejantes, el otro concebido dentro de un sistema CAD/CAM, dedicado a la fabricación de matrices para la inyección de suelas de hule.
\end{abstract}

\section{SUMMARY}

This work has the objective to bring a concise description ofthe postprocessor system funtion into a CAD/CAM system. Two different conception of postprocessors are showing; the first, a general kind oriented to use with APT or simmilar processors, and a second related to a dedicated CAD/CAM system used to design and machining ofshoe sole's injection rubber moulds.

\section{POSPROCESADORES}

La generación de programas de control numérico (NC) debe dividirse en dos etapas:

La primera produce las instrucciones geométricas para g!liar la herramienta y los parámetros de mecanizado como avance, rotaciones, etc. Esta información viene de un procesador general como por ejemplo el APT (Automatically Programmed Tools).

La segunda etapa es efectuada por el posprocesador, que cumple con la función de ligar el archivo generado por el procesador con la máquina NC. El posprocesador (Pp) tiene que "conocer" el controlador de la máquina para producir la pieza dentro de las tolerancias especificadas, con las velocidades establecidas y teniendo en consideración los efectos dinámicos del sistema. Es por esto que se requiere un Pp para cada combinación de controlador/máquina-herramienta. La ventaja de haber dividido el proceso en dos etapas radica en que los efuerzos para adaptar un Pp son menores de lo que sería necesario para modificar un programa mayor como el APT para cada controlador.

\subsection{ELEMENTOS DEL POSPROCESADOR}

La figura 1 presenta la estructura de un $\mathrm{Pp}$, seguidamente será dada una breve descripción de sus partes.

Entrada: el elemento de entrada hace la lectura de las informaciones surgidas a la salida del APT .

Movimiento: Esta es la parte principal del $\mathrm{Pp}$, la cual efectúa todas las instrucciones relacionadas con los movimientos de la herramienta. Incluye dos funciones:

La geométrica, que efectúa las conversiones de coordenadas de entrada en coordenadas propias de la máquina, cuidando de mantenerse dentro de las tolerancias, en caso de salirse de la tolerancia, vuelve a recalcular la transformación; también verifica que los desplazamientos no sobrepasen la capacidad de la máquina.

La función dinámica cuida que no ocurran sobrepasajes, con lo cual controla las distancias de aceleración y de desaceleración. 
Auxiliar: El elemento auxiliar compara las funciones preparatorias y misceláneas disponibles en el controlador de una configuración específica con las funciones requeridas, provenientes de la entrada, para así determinar cuándo son compatibles con el controlador de la máquina, en caso de ser así, son trasferidas al elemento de salida, de lo contrario habría un mensaje de error.

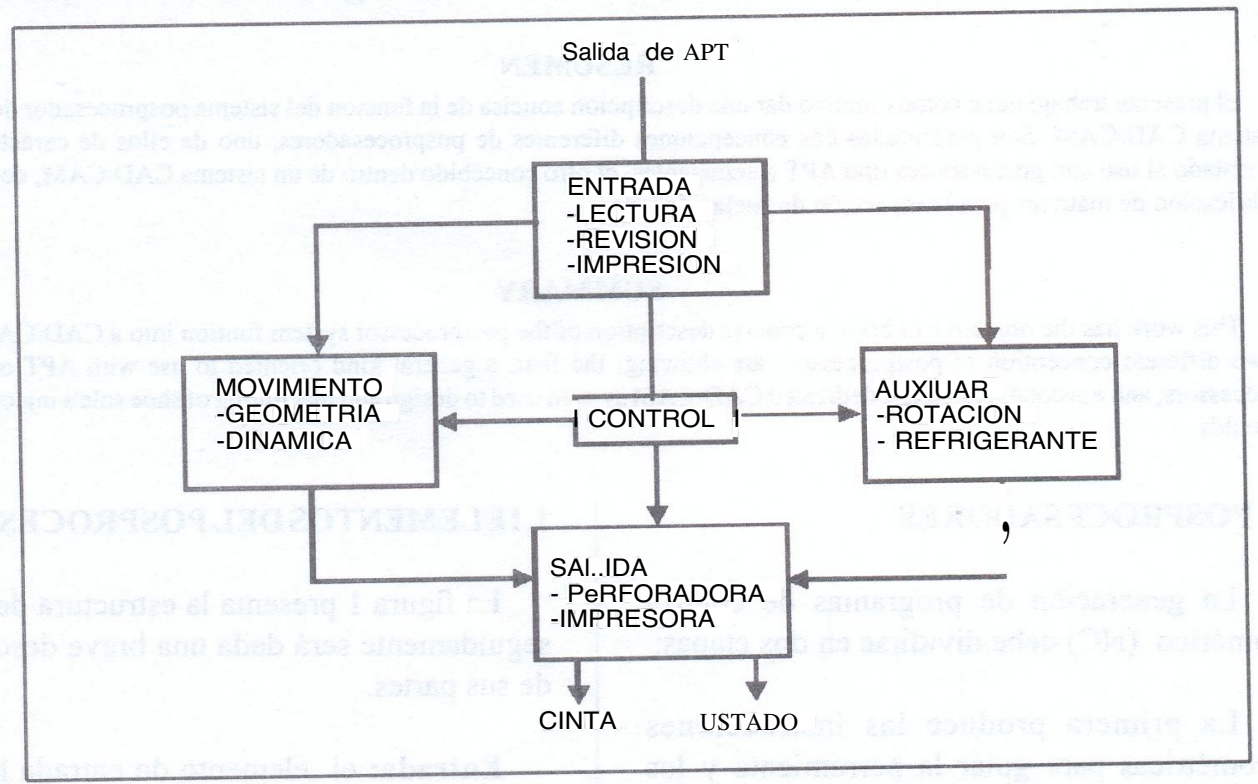

Figura 1 Estructura del posprocesador de un sistema CAD/CAM.

Salida: Es el elemento de salida que recibe la información de los elementos de movimiento y auxiliar, después es convertida en un apropiado patrón de comunicación compatible con el controlador de la máquina, y provee salidas tales como: la cinta perforada, el listado del programa o alguna otra similar.

Controlador: Es el elemento de control que genera la regulación de tiempo, permitiendo el flujo del programa, que a su vez controla también el flujo de datos de salida y la aceptación de nuevos datos para ser posprocesados.

\section{POSPROCESADOR \\ NECESIDAD}

MODULAR:

A medida que la programación de máquinas NC fue orientándose al uso de computadores, se fue mostrando la necesidad de un enfoque diferente de los Pp a fin de reducir su costo de desarrollo. Un estudio intensivo de los $\mathrm{Pp}$ dio como resultado una lista de funciones comunes' a todos ellos, entre las cuales se encuentran las siguientes:

1. Distribución de funciones auxiliares.

2. Procesador principal.

3. Def. de tolerancias dinámicas.

4. Plano de holgura.

5. Control de la mesa rotativa.

6. Establecimiento del centro de rotación.

7. Establecimiento del status del refrigerante.

8. Cálculo de la carrera máxima.

9. Rotación de coordenadas.

10. Traslación de coordenadas.

11. Limitación del avance en torno a una esquina. 
Para cada una de estas funciones es preparada una subrutina, la cual puede ser usada en cualquier $\mathrm{Pp}$, independientemente, del sistema de control (CNC). Así es establecida una biblioteca de "módulos". De esta forma es posible obtenerunpp con menos trabajo, siendo preciso crear únicamente una función "conductora" para el sistema máquinaherramienta/controlador.

La diferencia entre un pp modular y uno no modular está básicamente en la forma como ellos son ensamblados; sin embargo, el funcionamiento de ambos puede ser igual.

\section{DESARROLLO DE POSPROCESADORES}

EL desarrollo de pp requiere que los siguientes requisitos sean cumplidos:

- Conocimiento del lenguaje de alto nivel del sistema procesador.

- Conocimiento de la estructura del archivo CLDA TA, generado por el procesador.

- Conocimiento de un lenguaje de programación de alto nivel (FORTRAN, PASCAL, C, etc.).
- Conocimiento de las características de la máquina para la cual será desarrollado el sistema.

- Conocimiento de lenguaje de programación manual (NC).

- Conocimientos básicos de mecanizado.

\subsection{FLUJO BÁSICO DEL SISTEMA Pp.}

En la figura 2 se describe el flujo básico del sistema Pp.

El módulo de preparación tiene por finalidad administrar el proceso de lectura del archivo CLDA TAy la preparación del archivo intermediario (que contiene apenas los datos necesarios al pp, retirados del CLDATA).

El módulo de tratamiento/generación de reportes es responsable de:

- El análisis del archivo intermediario.

- La generación del programa NC.

- La generación de los reportes auxiliares.

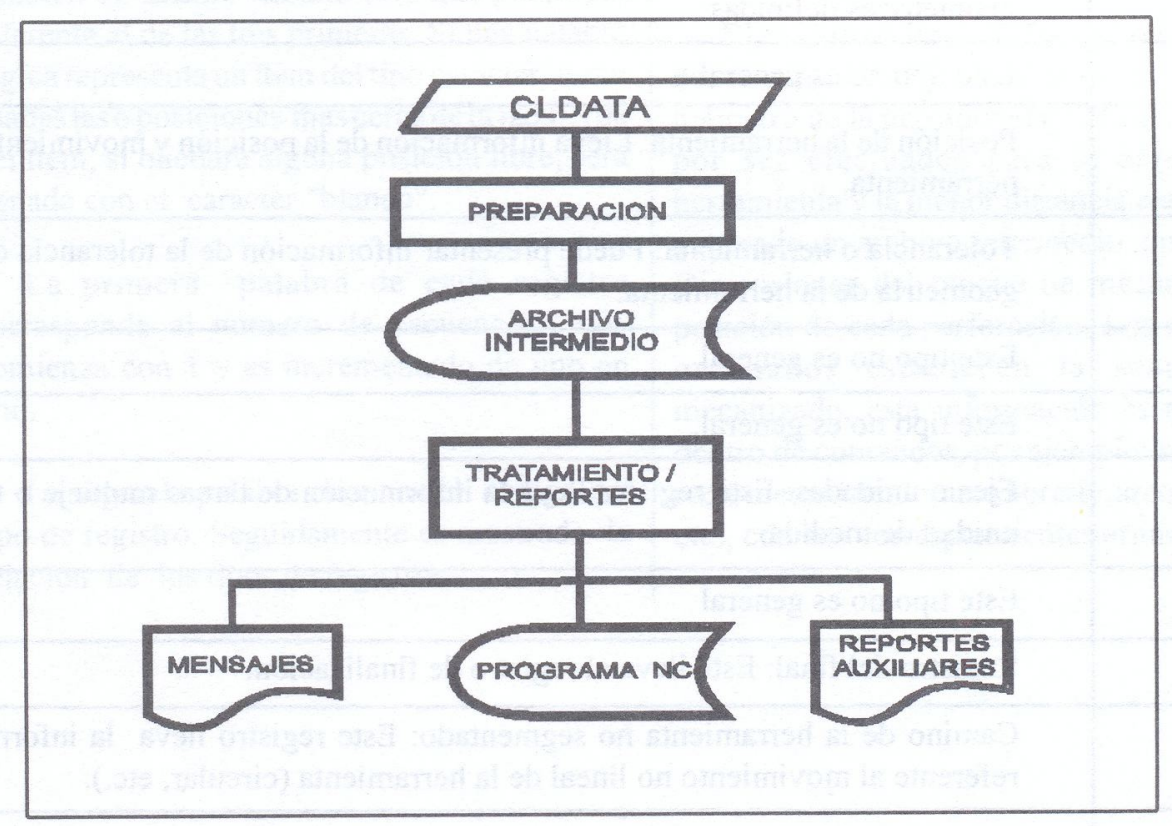

Figura 2 Diagrama de flujo del Pp. 


\subsection{ARCHIVOS UTILIZADOS y GENERADOS POR Pp}

El sistema pp utiliza el archivo CLDA TA generado por el procesador, este es un archivo binario cuyas características están especificadas en la norma ISO 3592-1978(E). De este archivo son extraídas las informaciones para la generación de programas NC.

Para trabajar las informaciones, el Pp crea un archivo de trabajo de acceso directo, un archivo temporario de cinta y el archivo de cinta propiamente dicho.

El archivo de trabajo de acceso directo mantiene las informaciones leídas del CLDA TA, incrementadas con otras que sean relevantes al tratamiento de cada comando del procesador (APT, EXAPT, etc). Existe una rutina que desvía el flujo del programa para el tratamiento de cada comando, con lo cual generada la información relevante al comando en la creación de las funciones del movimiento de la máquina. Estas informaciones son después almacenadas en el archivo de trabajo; el proceso es repetido hasta aparecer el último comando del programa (FINI).

El archivo temporal de cinta permanece temporalmente en disco y contiene el código NC generado por el Pp. Este archivo es, entonces, leído por la rutina de generación de archivos de cinta y copiado para otro archivo, considerando algunas funciones especiales del Pp (PPFUN o

\begin{tabular}{|c|c|}
\hline CODIGO & EXPLICACION \\
\hline 1000 & $\begin{array}{l}\text { Secuencia de entrada: Lleva la identificación de las declaraciones del programa } \\
\text { de la pieza. }\end{array}$ \\
\hline 2000 & Instrucciones del posprocesador: Lleva las instrucciones para el pp. \\
\hline 3000 & $\begin{array}{l}\text { Datos de la superficie: Lleva las definiciones canónicas de las superficies } \\
\text { geométricas definidas. }\end{array}$ \\
\hline 4000 & Este tipo no es general. \\
\hline 5000 & $\begin{array}{l}\text { Posición de la herramienta: Lleva información de la posición y movimiento de la } \\
\text { herramienta. }\end{array}$ \\
\hline 6000 & $\begin{array}{l}\text { Tolerancia o herramienta: Puede presentar información de la tolerancia o de la } \\
\text { geometría de la herramienta. }\end{array}$ \\
\hline 7000 & Este tipo no es general \\
\hline 8000 & Este tipo no es general. \\
\hline 9000 & $\begin{array}{l}\text { Ejes o unidades: Este registro lleva la información de tareas multieje o tipo de } \\
\text { unidad de medida. }\end{array}$ \\
\hline 12000 & Este tipo no es general \\
\hline 14000 & Registro del final: Este lleva el registro de finalización. \\
\hline 15000 & $\begin{array}{l}\text { Camino de la herramienta no segmentado: Este registro lleva la información } \\
\text { referente al movimiento no lineal de la herramienta (circular, etc.). }\end{array}$ \\
\hline 16000 & $\begin{array}{l}\text { Descripción de contornos de la pieza: Contiene las informaciones referentes a la } \\
\text { definición de contornos. }\end{array}$ \\
\hline 20000 & Comandos literales del Pp: Este registro lleva información literal para el Pp. \\
\hline
\end{tabular}


PPWORD) que alteran la distribución en la cinta perforada $o$ en el medio de salida que se esté usando.

\subsection{ESTRUCTURA DEL ARCHIVO CLDAT A (SEGÚN NORMA ISO 3592- 1978 (E) )}

El archivo CLDA TA consiste en un conjunto de registros lógicos organizados en bloques, reunidos en un medio físico. Los registros lógicos del CLDA TA son independientes del computador; sin embargo, los bloques físicos son dependientes del computador. La especificación del CLDA TA está basada en el lenguaje de procesamiento APT, no obstante, han sido dadas algunas consideraciones para extensiones tecnológicas.

Cada registro lógico consiste en una secuencia de palabras lógicas, que pueden llegar hasta 245; cada una de ellas puede representar un entero, un real o seis caracteres. Las primeras tres palabras de cada registro tienen el mismo tamaño y son siempre enteros, el resto tiene también el mismo tamaño solo que puede ser diferente al de las tres primeras. Si una palabra lógica representa un ítem del tipo carácter, serán usadas las 6 posiciones más cerca de la izquierda del ítem, si quedara alguna posición libre, será llenada con el carácter "blanco".

La primera palabra de cada registro corresponde al número de secuencia, que comienza con 1 y es incrementado de uno en uno.

La segunda palabra contiene el código del tipo de registro. Seguidamente es mostrada la relación de los tipos de registros.
4. SISTEMA CAD/CAM PARA GENE-

RACION DE MOLDES PARA

INYECTADO DE SUELAS DE HULE PARA ZAPATOS, OTRA PERSPECTIV A DEL POSPROCESADOR.

A continuación es descrito un sistema CAD/ CAM para generación de matrices de suelas, desarrollado por Orando y Delfino en. el ORUCON, Departamento de Ingeniería Mecánica, Universidad Federal de Santa Catarina en Brasil. El sistema consta de un conjunto de facilidades para el disefio de la geometría de la suela y herramientas que permiten crearla de manera gráfica. Esta información es despues procesada en el módulo CAM, con lo que se obtiene, a la salida, el programa $\mathrm{NC}$ requerido para ser usado en el centro de mecanizado en la fabricación de las cavidades del molde.

EL sistema CAM implementado tiene dos objetos computacionales.

\section{- Traductor \\ - Pos procesador}

El traductor lee la información topológica del sistema CAD y clasifica los elementos por diámetro de la herramienta, define los orificios por ser efectuados para la entrada de la herramienta y la menor distancia entre ellos. Es generado un archivo intermedio que contiene: Dimensiones del bloque de mecanizado y la posición de cada perforación. Los orificios así ordenados establecen la secuencia de mecanizado, esta información es almacenada dentro de comandos, por ejemplo: recta rápida, recta mecanizando, arco horario, arco antihorario etc., con sus correspondientes argumentos. 
El Pp toma los comandos y sus argumentos y va generando bloques de programación NC para una determinada máquina, esto es, asocia la información del archivo intermediario con las funciones preparatorias y auxiliares de la forma como ellas son "entendidas" por un determinado sistema CNC.

El sistema descrito tiene la peculiaridad de tener un dominio de operaciones de mecanizado limitado, lo cual reduce el tamaflo del procesador (Traductor), sin embargo, este sistema CAD/ CAM ha sido implantado usando la técnica de programación orientada por objetos, la cual ofrece ventajas tales como:

- Reusabilidad

- Flexibilidad

- Rapidez en el desarrollo

- Menor costo de desarrollo

Estas posibilitan su utilización en el desarrollo de otros sistemas, principio a que se hacía referencia al hablar del concepto modular.

\section{CONCLUSIONES}

Puede concluirse que ambos sistemas están fundamentados en los mismos principios de concepción modular, lo que facilita su adaptación a diferentes combinaciones de máquina- herramienta/controlador CNC. Además, se debe resaltar la importancia y la necesidad de tomar en cuenta este tipo de entidades en la selección de un sistema de CAD/CAM y en la selección de máquinas-herramienta de control numérico, para su adecuada adaptación a las instalaciones ya existentes.

\section{BIBLIOGRAFIA}

FOGA $<$;A CARNEIRO, MIRIAM .C. C. Tecnolo ía em desenvolvimento de pós-Processadores. Universidade Federal de Santa Catarina, GRUCON.

GRANDO, CLAUDIO; DELFINO C., RICARDO. Sistema para Geras:i'o de Matrizes de Solados Injetados. Universidade Federal de Santa Catarina, GRUCON, 1991.

INTERNA TIONAL ORGANIZA TION FOR STANDARIZATION (ISO), Numerical Control of Machines: ISO Standards Handbook 7. Ginebra, 1981.

KOREN, YORAM. Computer Control of Manufacturing Systems. McGraw Hill, SIngapore, 1986.

OLESTEN, NILS O. Numerical Control. Wiley Interscience, New York .. 